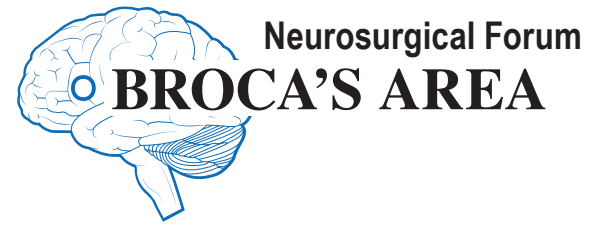

\section{Neurosurgery and the rise of academic social media: what neurosurgeons should know}

\author{
Joseph R. Linzey, BSc, ${ }^{1}$ Christopher S. Graffeo, MD, ${ }^{2}$ \\ Justin Z. Wang, MD, ${ }^{3}$ Ali S. Haider, BSc, ${ }^{4}$ and \\ Naif M. Alotaibi, MD, MSc ${ }^{3}$ \\ ${ }^{1}$ University of Michigan Medical School, Ann Arbor, Michigan; \\ 2Department of Neurologic Surgery, Mayo Clinic, Rochester, Minnesota; \\ ${ }^{3}$ Division of Neurosurgery, Department of Surgery, University of Toronto, \\ Ontario, Canada; and ${ }^{4}$ Texas A\&M University Health Science Center \\ College of Medicine, College Station, Texas
}

$\mathrm{S}$ OCIAL media has evolved to take on a major role in the personal and professional lives of people across the world. As of 2016, Pew Research Center data estimates that $86 \%$ of US adults used the Internet, of whom $79 \%, 24 \%, 32 \%$, and 29\% used Facebook, Twitter, Instagram, and LinkedIn, respectively. ${ }^{12}$ In parallel, social media use among clinicians, including neurosurgeons, neurosurgical departments, and neurosurgical journals, has increased exponentially over the past 3 years. ${ }^{2,4,6,10,11,19}$ Given this dramatic rise and the increasing emphasis being placed on social media in professional networking, patient recruitment and education, and the dissemination or discussion of new scientific knowledge, our objective was to highlight the potential benefits and risks of developing a social media presence for members of the neurosurgical community in particular, as well as offer a preliminary guide for the novice social media user.

\section{Getting Started}

Many social media platforms exist, and each serves a distinct purpose (Table 1). However, all social media platforms share the same, unique goal of disseminating information and allowing for the cultivation of connections and relationships that transcend the bounds of traditional geographic boundaries. Facebook and Twitter remain the most commonly used among academics. Both allow us- ers to post original content, including text, images, and video. While Facebook allows the text of a given post to be of any length, Twitter currently limits the text to 280 characters per "tweet." Facebook utilizes a "friend" system, which typically requires mutual agreement between users to allow shared viewing of posted content. Twitter, on the other hand, has a "follow" system, which allows a user to observe the activity of any other public Twitter account voluntarily, with no reciprocal follow or approval action required. In the academic context, this feature allows Twitter users to more readily expand their network by following and interacting with faculty and departments outside of their home institution.

Developing a professional social media presence on Twitter can be daunting; however, a few simple tips can smooth the process, even for technological neophytes (Fig. 1), for example, 1) using the same professional, easily recognizable picture across all social media sites; 2) beginning by following and interacting with large governing bodies like the AANS (@AANSNeuro) or publications like the Journal of Neuro-Oncology (@JNeurooncol); and 3) making a daily habit of interacting with at least one interesting piece of content as you review your feed. From there, building to the goal of generating 3-4 original posts per week while making sure to engage with the platform on a regular basis further establishes one's social media presence. While engagement can vary across users, an active account typically requires 10 or 15 minutes of attention per day, although this can be broken up throughout the day.

\section{Establishing a Narrative}

One of the greatest advantages of social media is the venue it provides for individual neurosurgeons or departments to develop and broadcast their unique professional narrative. By selecting the content that they share or interact with, each social media user may promote particular themes that are then relayed and reinforced to the academic community and public at large. In an era when the public is turning to the Internet and sites like HealthGrades. com for medical advice before selecting a physician, the 
TABLE 1. Examples of some of the most common social media platforms with a brief platform overview and example post for that platform

\begin{tabular}{|c|c|c|}
\hline Platform & Platform Overview & Example Post \\
\hline Twitter & $\begin{array}{l}\text { "Tweet" or share } 280 \text {-character messages with options to at- } \\
\text { tach images and videos. }\end{array}$ & Interesting \#cerebrovascular case via @TheJNS. \#neurosurgery \\
\hline Facebook & $\begin{array}{l}\text { Post messages of any length with options to attach images and } \\
\text { videos. }\end{array}$ & Clip vs coil for this aneurysm? What are your thoughts? \\
\hline Linkedln & $\begin{array}{l}\text { Professional networking site that can act like an online curricu- } \\
\text { lum vitae. Options are available to post text, image, or video } \\
\text { content as well. }\end{array}$ & $\begin{array}{l}\text { I am an expert in cerebrovascular neurosurgery. Below is a list of my } \\
\text { publications and endorsements. }\end{array}$ \\
\hline Instagram & Post images and videos with text captions. & $\begin{array}{l}\text { Here is a picture of a basilar apex aneurysm after Y-stent assisted } \\
\text { coiling. }\end{array}$ \\
\hline YouTube & Repository of videos that users can upload and share. & $\begin{array}{l}\text { This is a video demonstrating the ideal technique for clipping a } \\
\text { middle cerebral artery aneurysm. }\end{array}$ \\
\hline Snapchat & $\begin{array}{l}\text { Short, transient video clips, for public or private transmission, } \\
\text { that are deleted after } 24 \text { hours or once viewed. }\end{array}$ & $\begin{array}{l}\text { Having a great time at the \#AANS2017 opening reception with all my } \\
\text { trail buddies! }\end{array}$ \\
\hline Blogger & Website used to write longer, more thoughtful posts or blogs. & $\begin{array}{l}\text { Below is a step-by-step description of the operative techniques I } \\
\text { used for this bypass. }\end{array}$ \\
\hline
\end{tabular}

ability to solidify and directly influence an online presence and narrative is imperative.

An important aspect of this concept is applicable to residents or junior faculty, who can utilize social media to emphasize their specific interests within neurosurgery, and establish themselves prominently in the online discussion of key topics among their colleagues or thought leaders in the field. This may be of particular interest to techsavvy younger trainees, as the academic use of social media is still in its relative infancy, and therefore becoming a reliable and highly utilized information source within a specific niche of neurosurgery is accessible and powerful. By posting de-identified, intraoperative images and videos, or high-quality imaging studies, or interesting learning cases, young faculty and residents can quickly amass a significant social media following of eager learners, ranging from interested medical students and residents, to faculty colleagues at other institutions, or even the lay public and disease advocacy groups. The Young Neurosurgeons Committee (YNC; @youngneuros) has been exemplar in its efforts to utilize social media to facilitate networking opportunities between junior and senior faculty members and ease the difficult transition from resident to attending status.

The tactic of targeted development can be further enhanced by tailoring posts to the interests of the intended audience or creating content to interact with a specific group of patients, colleagues, or organizations. Such an approach is readily optimized using a wide range of data analytics, such as user demographics or click-through rates on various posts or content categories. For example, the neurosurgical department at the University of Toronto published the results of their early social media engagement efforts, which demonstrated a base of predominantly male followers, aged 18-34 years, who were most likely to interact with posts containing images, videos, or mentions of senior faculty. These analytics may be similarly used to increase reach to a specific audience to expand one's target demographic. ${ }^{4}$

\section{Education}

Residents have long been the educational center of most neurosurgical departments, journals, and academic mentors, all of whom are increasingly mobilizing digital resources for teaching. As an example of adopting a key component of neurosurgical training into the digital world, online journal clubs are being carried out over social media platforms. These events generally begin with a social media announcement heralding the anticipated date, time, and articles to be discussed. Interested students, residents, and faculty members can then log in from anywhere in the world to go over the papers, discuss salient clinical issues, or simply network with one another. These interactions foster an environment of collaboration between institutions, fostering the free exchange of ideas, and emphasizing the value of an open, collaborative enterprise between residents and programs. In fact, in an effort to continually improve resident education, the Congress of Neurological Surgeons (@CNS_Update) has recently introduced monthly journal club podcasts through their journal Neurosurgery (@NeurosurgeryCNS).

One idiosyncratic but useful feature of social media pertinent to online journals and journal clubs is the use of hashtags: a keyword preceded by the pound sign (e.g., \#neurosurgery), as a way of marking or categorizing content. These may signal a topic of interest (e.g., \#cerebrovascular, \#spine), or a case for review (\#ecase), while others may generally identify resources geared toward students and/or residents (\#FOAM or \#FOAMedEd, short for Free Open Access Medicine/Medical Education). Of particular interest, \#FOAM resources like The Neurosurgical Atlas - a copartner of the JNSPG and AANS-have become popular among trainees and have utility throughout residency, as preliminary training resources to new interns, as refreshers for individuals preparing for cases, and/or as study material for the board exams.

As hashtags gain prominence and become more standardized, they have begun to prove useful both in research-identifying key articles for review, or providing rapid content indices-and in continuing medical educa- 
tion, with new findings on common topics often circulating widely, and undergoing extensive discussion by experts worldwide. This active, ongoing discourse highlights another critical educational benefit of trainee involvement with social media: engagement. Anecdotally, many residents and junior staff who maintain an active and interactive social media presence, including the authors of this review, cite social media as a driving force in their daily or weekly reading, and an easy, accessible way to stay on top of new discoveries and their reception by the neurosurgical or neuroscientific community and the public.

In the spirit of many other \#FOAM resources, a number of journals and institutions are aiming to actively promote this type of serial engagement, using "interesting case" series, discussion of clinically minded charts and utilities, and/or integration of online events into weekly or monthly publication cycles. Similarly rare, complex, or challenging cases are frequently promoted and discussed, providing a source of additional exposure to residents, who otherwise might only see certain entities a few times throughout their entire training, and can now watch and learn from a range of related operations with walkthroughs and discussions of pitfalls or complications. ${ }^{16}$ For example, the American Journal of Neuroradiology (@TheAJNR) has a running interesting case series to highlight a number of rare and bread-and-butter cases within the sphere of neuroradiology.

\section{Patient Education}

As Internet access and social media use become increasingly integrated into the daily life of the public, online resources are becoming a more critical and utilized educational tool that patients actively seek out and engage with regarding their healthcare concerns, sometimes before even coming to medical attention. The ways in which specific patient groups tend to engage with social media speaks to their needs as a group and how these platforms can be mobilized to better serve them worldwide. In a recent study focused on the use of social media in subarachnoid hemorrhage and brain aneurysms, several key, interrelated themes emerged. Across dozens of groups and pages on Facebook and Twitter, about $28 \%$ of posts and comments revolved around inspiration and motivation, $26 \%$ provided or shared information, $14 \%$ requested disease information, $12 \%$ sought emotional support, $8 \%$ discussed admiration, and $8 \%$ expressed loss and grief. ${ }^{5}$ Similar studies have been done analyzing how social media is used by patients with hydrocephalus, epilepsy, and traumatic brain injury. ${ }^{1,8,14,18}$ Collectively, these studies demonstrate that social media has the potential to fill a profound need for connection, community, and understanding by others in similar situations. Consequently, endorsing and empowering the creation and evolution of these communities may be the single most impactful social media enterprise that the neurosurgical community can take up on behalf of our patients.

\section{Dissemination of Science}

In so far as the propagation of new neurosurgical knowledge via social media is empowering to residents and students who choose to engage with it actively, so also has

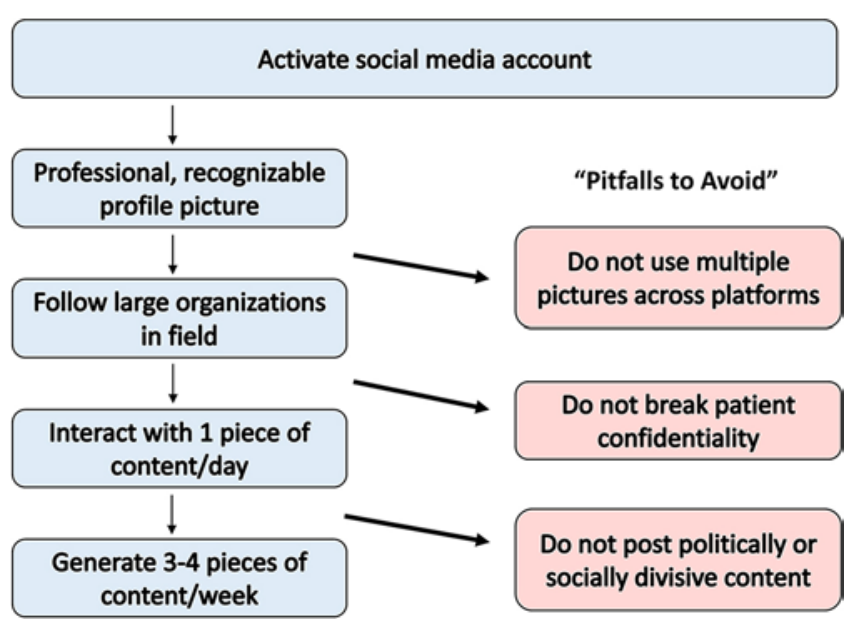

FIG. 1. A quick-start guide to begin to develop a social media presence. Common but preventable pitfalls are also included. Figure is available in color online only.

social media provided the opportunity for researchers and thought leaders to expand their reach, promote their ideas, and use either professional discourse or crowd-sourced opinions to help brainstorm, refine, or otherwise advance their scientific careers. This speaks to the underlying mission of scientific discovery, particularly by providing a mechanism for individual researchers to, in part, transcend the pressure to simply publish extensively. Instead, they can become researchers who are not simply prolific in their number of publications, but also whose work is more broadly read, discussed, cited, and debated. The achievement of this goal depends in large part on the visibility of their work and the effective promotion of their ideas and accomplishments to others within the field through social media. Eysenbach diagrammed this potential relationship between social media impact and research impact (Fig. 2). ${ }^{9}$

Objective measures of individual academic success have thus far been largely determined by bibliometric indices, including but not limited to the h-index, m-index, g-index, e-index, or i10-index. ${ }^{15}$ However, these metrics do not completely account for the rising prevalence of digital publications, which required the development of an alternative scale. In 2010, Altmetrics (https://www.altmetric. com) was proposed as an article scoring system that determined impact based on a number of additional metrics, including views and downloads from the journal site (or a third-party distributor, such as PubMed Central), bookmarks in CiteULike or shares in Mendeley, mentions in prominent blogs or Wikipedia articles, and shares on social media platforms. ${ }^{13}$

A recent study analyzed 5794 neurosurgery articles and found that the average Altmetric Attention score was 4.7 $\pm 22.4 .{ }^{17}$ Notably, articles that were published in journals with an active social media presence had significantly higher Altmetric Attention scores-perhaps the first objective data point demonstrating the role that active social media engagement can play in expanding an article's reach. ${ }^{17}$ By extension, traditional bibliometrics also benefit indirectly from social media promotion, given that the more individuals and institutions read, engage with, or simply become aware of a new publication, the more 


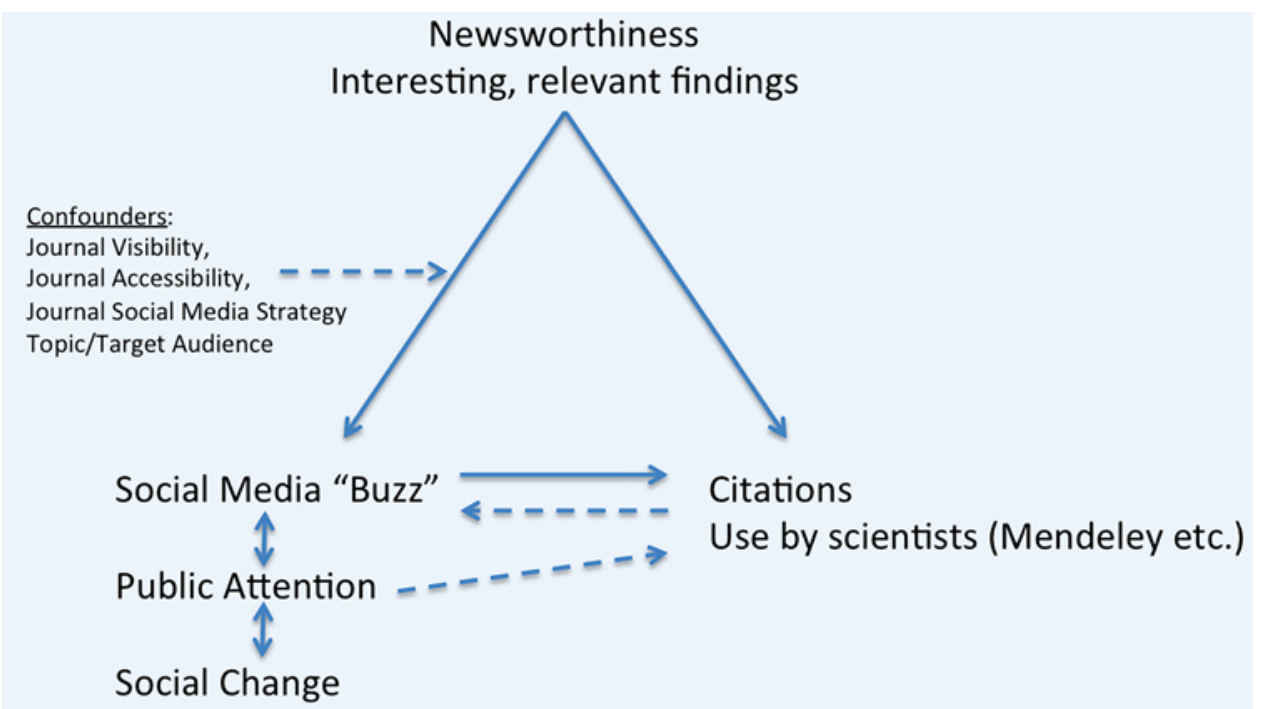

Social Impact Metrics $\quad$ Research Impact Metrics

FIG. 2. Schematic of interplay between the impact of social media and the impact of research bibliometrics. First published in the Journal of Medical Internet Research (Eysenbach G: Can tweets predict citations? Metrics of social impact based on Twitter and correlation with traditional metrics of scientific impact. J Med Internet Res 13(4):e123, 2011). Copyright Gunther Eysenbach. CC BY 2.0 (http://creativecommons.org/licenses/by/2.0/). Figure is available in color online only.

likely they are to cite it themselves in the future. This selfevident conclusion has also been ratified objectively, with multiple reports demonstrating a significant association between a robust social media presence and academic productivity among major academic centers..$^{2-4,7}$ Even within the specific niche of academic neurosurgery, a preceding analysis identified the presence of a departmental social media account as significantly associated with higher academic impact scores among the faculty. ${ }^{3}$

\section{Future Directions}

The importance of social media as a vital, dynamic means for members of the neurosurgical community to establish and promote their unique narratives; participate in the education of students, residents, and patients; and disseminate their research discoveries is anticipated to substantially increase. Additionally, as more neurosurgeons, departments, and journals engage with social media, the quality of the educational content and patient resources will continue to improve, providing further positive feedback into the evolution of this exciting movement. Online resources like The Neurosurgical Atlas are revolutionizing neurosurgical education on many levels. Not only do online institutions like the Atlas provide high-quality images, videos, and commentary for learners to immerse themselves in, they allow them to do so at their leisure and at minimal cost.

These benefits are multiplied when the global neurosurgery community is considered, both in terms of providing training resources to remote locations and presenting content on how to manage unique cases or complications that are more likely to arise in certain regions, yet remain salient to any neurosurgeon. The development and integra- tion of a wide swath of other technologies into the sphere of neurosurgical social media is imminent, and tools like virtual reality, 3D printing, mobile-optimized content, and others will continue to advance the potential for social media to make major contributions to neurosurgical education. Already, live operations can be shared between centers via videoconferencing tools, allowing in-the-moment consultations or teaching opportunities to be transported across the globe, and one can imagine a future in which neurosurgeons in geographically disparate areas are able to collaborate in a single operation using robotics.

\section{A Cautionary Word}

As we have described in detail, social media offers a wide range of potential benefits for neurosurgeons interested in expanding their reach and contributing to the enterprise of digital education. However, as with any new technique or technology, there are attendant risks that should be treated with the utmost seriousness. Most importantly, the patient protections provided by the Health Insurance Portability and Accountability Act must be universally respected, and, correspondingly, all images, videos, or other potential personal health information must be completely de-identified. The ethics surrounding the production of operative videos and live videoconferencing should always be considered to protect patient privacy and ensure that we maintain the confidence entrusted to us by our patients.

Similarly, the seemingly innocuous interfaces through which we engage with social media-phone apps, Web pages, and other anonymous and personal technology-obscure the fact that publicly posted information has the capacity to reach a staggeringly large audience and, in many circumstances, cannot be rescinded. As such, the same 
professionalism that one would bring to the clinic or the stage of a national meeting is imperative, and a focus on objective, neurosurgical topics is encouraged, to avoid misrepresentation of oneself or one's institution. More specifically, topics and posts that are inherently political, socially contentious, or overtly divisive are strongly discouraged, as they have the potential to damage not only one's personal reputation but potentially also the doctor-patient relationship at large.

Even when posting on niche topics unlikely to be engaged with outside the neurosurgical community, thoughtful attention to detail is recommended. Studies and articles should be read carefully before public comment, regardless of one's intention to take a positive or negative tone. If one feels compelled to offer a well-reasoned critique, or contribute to the discourse on a neurosurgical debate, he/she has to focus on the scientific content and distance himself/herself from any personal or professional biases. Although the general experience of neurosurgeons with social media has been overwhelmingly positive, the relative number of neurosurgeons on social media remains small and the potential for poor judgment is not negligible; notwithstanding, like common intraoperative errors, most potential pitfalls are avoidable, provided that users adhere to the core principles of professionalism.

Finally, it should be noted that while social media can assist in fostering relationships and disseminating research, it should never become a substitute for in-person relationships and the production of original, academically rigorous science. Social media is best thought of as an additional tool in a neurosurgeon's armamentarium rather than the primary method of engaging with the scientific community.

\section{Conclusions}

Social media is increasing in popularity among academic neurosurgeons and neurosurgical trainees. If used properly and responsibly, social media can allow a young neurosurgeon to craft and share his/her professional narrative; participate in the education of medical students, residents, and patients; and develop a forum for disseminating scientific research, in order to both reach a wider readership and participate in distributed collaboration with like-minded colleagues in distant locations. Perhaps most importantly, social media within the neurosurgical community has thus far appeared to evolve through cycles of self-improving positive feedback, with the quality of posts and discussions improving over time and with increasing usership, a promising pattern for the future of digital neurosurgical education and research.

\section{References}

1. Ali R, Elsayed M, Kaur M, Air E, Mahmood N, Constantinou J, et al: Use of social media to assess the effectiveness of vagal nerve stimulation in Dravet syndrome: a caregiver's perspective. J Neurol Sci 375:146-149, 2017

2. Alotaibi NM, Badhiwala JH, Nassiri F, Guha D, Ibrahim GM, Shamji MF, et al: The current use of social media in neurosurgery. World Neurosurg 88:619-624, 624.e1-624.e7, 2016

3. Alotaibi NM, Guha D, Fallah A, Aldakkan A, Nassiri F, Badhiwala JH, et al: Social media metrics and bibliometric profiles of neurosurgical departments and journals: is there a relationship? World Neurosurg 90:574-579, 579.e1-579.e7, 2016

4. Alotaibi NM, Samuel N, Guha D, Nassiri F, Badhiwala JH, Tam J, et al: Social media for academic neurosurgical programs: the University of Toronto experience. World Neurosurg 93:449-457, 2016

5. Alotaibi NM, Samuel N, Wang J, Ahuja CS, Guha D, Ibrahim GM, et al: The use of social media communications in brain aneurysms and subarachnoid hemorrhage: a mixed-method analysis. World Neurosurg 98:456-462, 2017

6. Ban VS, Lega B, Batjer HH: Maximizing the potential of social media and social networks in neurosurgery. World Neurosurg 91:609-610, 2016

7. Bean JR: Academic output and social media: a marriage of opposites. World Neurosurg 90:651-653, 2016

8. Elkarim GA, Alotaibi NM, Samuel N, Wang S, Ibrahim GM, Fallah A, et al: Social media networking in pediatric hydrocephalus: a point-prevalence analysis of utilization. J Neurosurg Pediatr 20:119-124, 2017

9. Eysenbach G: Can tweets predict citations? Metrics of social impact based on Twitter and correlation with traditional metrics of scientific impact. J Med Internet Res 13:e123, 2011

10. Fargen KM, Ducruet AF, Hyer M, Hirsch JA, Tarr RW: Expanding the social media presence of the Journal of Neurointerventional Surgery: editor's report. J Neurointerv Surg 9:215-218, 2017

11. Fargen KM, Wilson TA, de Leacy R, Jadhav AP, Ducruet AF: Social media and JNIS: expanding the digital clique. J Neurointerv Surg 9:913-914, 2017

12. Greenword S, Perrin A, Duggan M: Social media update 2016. Pew Research Center. November 11, 2016. (http:// www.pewinternet.org/2016/11/11/social-media-update-2016/) [Accessed March 28, 2018]

13. Melero R: Altmetrics-a complement to conventional metrics. Biochem Med (Zagreb) 25:152-160, 2015

14. Meng Y, Elkaim L, Wang J, Liu J, Alotaibi NM, Ibrahim GM, et al: Social media in epilepsy: a quantitative and qualitative analysis. Epilepsy Behav 71 (Pt A):79-84, 2017

15. Prabhu VC, Swong K, Hendler G: Publication metrics in neurosurgery. World Neurosurg 105:993-996, 2017

16. Samuel N, Alotaibi NM, Lozano AM: YouTube as a source of information on neurosurgery. World Neurosurg 105:394398, 2017

17. Wang J, Alotaibi NM, Ibrahim GM, Kulkarni AV, Lozano AM: The spectrum of Altmetrics in neurosurgery: the top 100 "trending" articles in neurosurgical journals. World Neurosurg 103:883-895, 895.e1, 2017

18. Workewych AM, Ciuffetelli Muzzi M, Jing R, Zhang S, Topolovec-Vranic J, Cusimano MD: Twitter and traumatic brain injury: a content and sentiment analysis of tweets pertaining to sport-related brain injury. SAGE Open Med 5:2050312117720057, 2017

19. Zusman EE: Social media in academic neurosurgery. World Neurosurg 91:606-608, 2016

\section{Disclaimer}

All authors serve as members of the Social Media Team for the Journal of Neurosurgery Publishing Group (JNSPG). JNSPG had no role in study design, data collection and analysis, decision to publish, or preparation of the manuscript for submission.

\section{Disclosures}

The authors report no conflict of interest.

\section{Correspondence}

Naif M. Alotaibi: naif.alotaibi@mail.utoronto.ca. 